\title{
TWO LIGAND OXIDIO-VANADIUM(IV) COMPLEXES AS NOVEL EFFICIENT CATALYSTS IN MULTICOMPONENT REACTIONS FOR SYNTHESIS OF TETRAHYDROBENZOPYRAN DERIVATIVES
}

\author{
Mehdi Abaszadeh $^{1,2^{*}}$, Mohammad Seifi ${ }^{3}$ and S. Yousef Ebrahimipour ${ }^{4,5}$ \\ ${ }^{1}$ Pharmaceutics Research Center, Institute of Neuropharmacology, Kerman University of \\ Medical Sciences, Kerman 76175493, Iran \\ ${ }^{2}$ Department of Medicinal Chemistry, Faculty of Pharmacy, Kerman University of Medical \\ Sciences, Kerman, Iran \\ ${ }^{3}$ Department of Chemistry, Faculty of Sciences, Najafabad Branch, Islamic Azad University, \\ Najafabad, Esfahan, Iran \\ ${ }^{4}$ Department of Chemistry, Shahid Bahonar University of Kerman, Kerman 76169-14111, Iran \\ ${ }^{5}$ Department of Chemistry, Payame Noor University (PNU), 19395-4697 Tehran, Iran
}

(Received September 23, 2015; revised June 26, 2016)

\begin{abstract}
Two ligand oxido-vanadium(IV) complexes, [VO(L)(bipy)] (I) and [VO(L)(phen)] (II); $\left[\mathrm{H}_{2} \mathrm{~L}\right.$ : 4-bromo-2-(((5-chloro-2-hydroxyphenyl)imino)methyl)phenol, bipy: 2,2'-bipyridine and phen: 1,10phenanthroline], have been synthesized and characterized by elemental analysis, FT-IR, UV-Vis and conductivity measurements. These complexes, as new catalysts, were also used to synthesize the tetrahydrobenzopyran derivatives by three-component reaction of cyclic $\beta$-dicarbonyl compounds, malononitrile and aromatic aldehydes, in $\mathrm{EtOH}$, at reflux. Inexpensiveness, stability and the potential of being easily obtained can be noted as preponderance of these catalysts. Furthermore, high conversions, short reaction times and cleaner reaction profiles, are some of the advantages of this method. These catalysts can be recovered and reused several times without loss of activity.
\end{abstract}

KEY WORDS: Oxido-vanadium(IV) complex, Tetrahydrobenzopyran derivatives, Three-component reaction, Cyclic $\beta$-dicarbonyl compounds, Malononitrile

\section{INTRODUCTION}

The development of environmental safe and clean synthetic procedures has become the goal of present day organic synthesis. More than ever, the industry requests organic chemists the expansion of new strategies and technologies, to gain novel compounds in a fast, convergent and efficient way. One of the most attractive apparatus in modern organic chemistry is the use of multicomponent reactions (MCRs), which combine in one-pot at least three simple building blocks, provide a most powerful to make functionalized compounds [1-7]. MCRs as efficient and effective methods are considered a vital technique in the synthesis of many important heterocyclic compounds such as tetrahydrobenzopyran derivatives nowadays.

Tetrahydrobenzopyrans have recently attracted attention as an important class of heterocyclic compounds in the field of drugs and pharmaceuticals due to their useful biological and pharmacological properties. These compounds are widely used as anticancer [8], antimalarial [9], antileishmanial [10], antibacterial [11], antifungal [12], antitumor [13], antianaphylactic [14], antiallergenic [15], diuretic [16] and hypotensive [17] agents. They can also be used as cognitive enhancers for the treatment of neurodegenerative disease as Alzheimer's disease, amyoprophic lateral sclerosis, Huntington's disease, Parkinson's disease, AIDS associated [18].

Due to the important properties of tetrahydrobenzopyran derivatives, considerable attentions have been focused on the development of environmentally friendly procedures to synthesize

*Corresponding author. E-mail: abaszadeh@kmu.ac.ir 
tetrahydrobenzopyrans, by three-component reaction cyclic $\beta$-dicarbonyl compounds, malononitrile and aromatic aldehydes. Various catalytic systems such as potassium phthalimide$\mathrm{N}$-oxyl (POPINO) [19], $\mathrm{CaCl}_{2}$ under ultrasonic irradiation [20], high surface area $\mathrm{MgO}$ [21], tetrabutylammonium bromide [22], Nano- $\mathrm{ZnO}$ [23], $\mathrm{K}_{3} \mathrm{PO}_{4}$ [24], $\mathrm{NaOCl}$ under grinding [25], $\mathrm{SiO}_{2}$ nanoparticles [26], triethylbenzylammonium chloride (TEBA) [27] and $\mathrm{Ni}\left(\mathrm{NO}_{3}\right)_{2} \cdot 6 \mathrm{H}_{2} \mathrm{O}$ [28] have been reported. Each of these methods has its own merits, but the use of toxic organic solvents, expensive catalysts, containing transition metals, difficult work up, high reaction time, and low yields are drawbacks of these procedures. Thus to expand a simple and green synthesis of tetrahydrobenzopyran derivatives it is necessary to remove these restrictions.

In the present work, we have focused on the preparation and characterization of two new ligand oxidio-vanadium (IV) complexes containing the Schiff base 4-bromo-2-(((5-chloro-2-hyd roxyphenyl)imino)methyl)phenol $\left(\mathrm{H}_{2} \mathrm{~L}\right)$ and appropriate aromatic heterocyclic bases $(1,10$ phenanthroline and 2,2'-bipyridine). Moreover, in continuation of our studies on the synthesis of heterocyclic compounds by three-component reaction [29-32], the catalytic activity of these complexes was also investigated for multi-component reaction of dimedone (1a) or 1,3cyclohexanedione (1b), malononitrile (2) and aromatic aldehydes (3a-r) in ethanol at reflux (Scheme 1) to remove the above mentioned drawbacks.

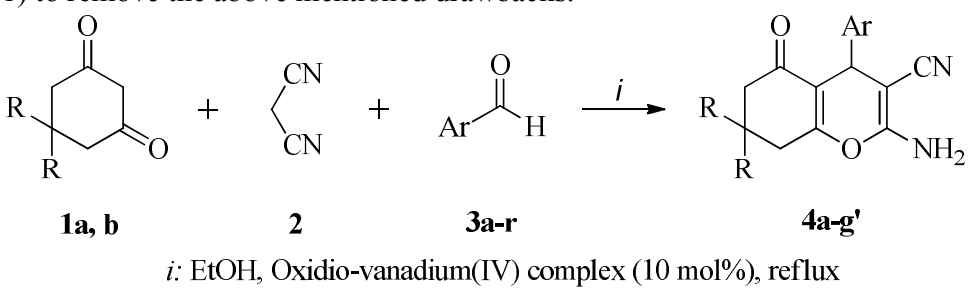

Scheme 1. Schematic diagram for synthesis of tetrahydrobenzopyran derivatives.

Herein we consider the synthesis of compound 2-amino-7,7-dimethyl-5-oxo-4-phenyl5,6,7,8-tetrahydro- $4 H$-chromene-3-carbonitrile (4a) for a representative example to show the advantage of this work in comparison with previously reported procedures. As shown in Table 1, our catalyst produces compound $\mathbf{4 a}$ with high yield in a short time. Also oxidio-vanadium(IV) complexes are a recyclable catalyst and ethanol was used as a green solvent. Moreover oxidiovanadium(IV) complexes are more stable in air and non-toxic compared with other catalysts.

Table 1. Comparison results of oxidio-vanadium(IV) complexes with other catalysts reported in the literature for the synthesis of compound $4 \mathbf{a}$.

\begin{tabular}{|c|l|l|c|c|c|}
\hline Entry & Catalyst & Condition & Recyclability & Time (min) & Yield (\%) \\
\hline 1 & Potassium phthalimide- $N$-oxyl & $\mathrm{H}_{2} \mathrm{O}$, reflux & No & 15 & $95[19]$ \\
\hline 2 & $\mathrm{CaCl}_{2}$ & EtOH, ultrasonic & No & 8 & $96[20]$ \\
\hline 3 & High surface area $\mathrm{MgO}$ & $\mathrm{H}_{2} \mathrm{O}$, EtOH,reflux & No & 27 & $94] 21]$ \\
\hline 4 & Tetrabutylammonium bromide & EtOH, reflux & No & 20 & $92[22]$ \\
\hline 5 & $\mathrm{Nano}_{2} \mathrm{ZnO}$ & $\mathrm{H}_{2} \mathrm{O}, 80{ }^{\circ} \mathrm{C}$ & No & 30 & $90[23]$ \\
\hline 6 & $\mathrm{~K}_{3} \mathrm{PO}_{4}$ & EtOH, stirred, r.t. & No & 45 & $94[24]$ \\
\hline 7 & $\mathrm{NaOCl}_{8}$ & Grinding & No & 15 & $80[25]$ \\
\hline 8 & $\mathrm{SiO}_{2}$ nanoparticles & EtOH, reflux & No & 25 & $94[26]$ \\
\hline 9 & Triethylbenzylammonium chloride & $\mathrm{H}_{2} \mathrm{O}, 90^{\circ} \mathrm{C}$ & No & 240 & $90[27]$ \\
\hline 10 & $\mathrm{Ni}\left(\mathrm{NO}_{3}\right)_{2} \cdot 6 \mathrm{H}_{2} \mathrm{O}$ & $\mathrm{H}_{2} \mathrm{O}$, reflux & No & 20 & $95[28]$ \\
\hline 11 & {$[\mathrm{VO}(\mathrm{L})($ phen $)]$} & EtOH, reflux & Yes & 20 & $90[$ This work] \\
\hline
\end{tabular}




\section{EXPERIMENTAL}

All chemicals and solvents were purchased from Aldrich and Merck and used without further purification. $\left[\mathrm{H}_{2} \mathrm{~L}\right]$ and $[\mathrm{VO}(\mathrm{L})($ bipy)] were prepared according to our previous work [33, 34]. Melting points were measured on an Electrothermal-9100 apparatus and are uncorrected. IR spectra were recorded on a Brucker FT-IR Tensor 27 infrared and FT-IR 8400-Shimadzu spectrophotometer. ${ }^{1} \mathrm{H}$ NMR spectra were recorded on a Brucker Avance III $400 \mathrm{MHz}$ spectrometer. ${ }^{13} \mathrm{C}$ NMR spectra were recorded on the same instruments at $100 \mathrm{MHz}$ using TMS as an internal standard. Molar conductance measurements were made by means of a Metrohm 712 conductometer in DMSO. Elemental analyses were performed using a Heracus CHN-ORapid analyzer and Thermo Finnigan Flash Elemental Analyzer 1112EA. Electronic spectra of the complexes in DMSO solutions were recorded with a Shimadzu model 2550 UV-Vis spectrophotometer.

General procedure for preparation of the complexes

A $8 \mathrm{~mL}$ methanolic solution of $\mathrm{H}_{2} \mathrm{~L}(0.1 \mathrm{mmol}, 0.03 \mathrm{~g})$ and equimolar quantity of $\mathrm{VOSO}_{4} \cdot 3 \mathrm{H}_{2} \mathrm{O}$ $(0.03 \mathrm{~g})$ was refluxed for $1 \mathrm{~h}$. Appropriate aromatic heterocyclic base $(0.1 \mathrm{mmol}, 0.02 \mathrm{~g})$ was added to the resulting brown solution, and the reflux was continued for $3 \mathrm{~h}$. After cooling, the formed solid was filtered off, washed with cold ethanol, and dried in a vacuum desiccator over $\mathrm{CaCl}_{2}$.

(2,2'-Bipyridine) [4-bromo-2-((5-chloro-2-hydroxyphenylimino)methyl)phenol] oxido-vanadium (IV) [VOL(bipy)] (I). Yield: $67 \%$. m.p.: $236{ }^{\circ} \mathrm{C}$. Molar conductivity $\left(1.0 \times 10^{-3} \mathrm{M}\right.$, DMSO $): 21.5$ $\Omega^{-1} \mathrm{~cm}^{2} \mathrm{~mol}^{-1}$. Anal. calc. for $\mathrm{C}_{23} \mathrm{H}_{15} \mathrm{BrClN}_{3} \mathrm{O}_{3} \mathrm{~V}$. $\mathrm{CH}_{3} \mathrm{OH}\left(579.72 \mathrm{~g} \mathrm{~mol}^{-1}\right): \mathrm{C}, 49.72 ; \mathrm{H}, 3.30 ; \mathrm{N}$, 7.25. Found: C, 49.68; H, 3.22; N, 7.31\%. FT-IR (KBr), $\mathrm{cm}^{-1}: v(\mathrm{CH}) 2855-3048, v(\mathrm{C}=\mathrm{N}) 1597$, $v\left(\mathrm{C}=\mathrm{C}_{\text {ring }}\right) 1512, v(\mathrm{C}-\mathrm{O}) 1288, v(\mathrm{~V}=\mathrm{O}) 948, v(\mathrm{C}-\mathrm{Cl}) 702, v(\mathrm{C}-\mathrm{Br}) 648$. UV/Vis (DMSO), $\lambda_{\max }$, $\mathrm{nm}\left(\log \varepsilon, \mathrm{L} \mathrm{mol}^{-1} \mathrm{~cm}^{-1}\right): 276$ (4.5), 444 (4.11), 658 (2.1).

(1,10-Phenanthroline) [4-bromo-2-((5-chloro-2-hydroxyphenylimino)methyl)phenol]oxidovanadium(IV) [VOL(phen)] (II). Yield: $71 \%$. m.p.: $253{ }^{\circ} \mathrm{C}$. Molar conductivity $\left(1.0 \times 10^{-3} \mathrm{M}\right.$, DMSO): $28.2 \Omega^{-1} \mathrm{~cm}^{2} \mathrm{~mol}^{-1}$. Anal. calc. for $\mathrm{C}_{25} \mathrm{H}_{15} \mathrm{BrClN}_{3} \mathrm{O}_{3} \mathrm{~V} . \mathrm{H}_{2} \mathrm{O}\left(589.72 \mathrm{~g} \mathrm{~mol}^{-1}\right)$ : C, 50.92 ; H, 2.91; N, 7.13. Found: C, 50.37; H, 2.82; N, 7.18\%. FT-IR (KBr), $\mathrm{cm}^{-1}: v(\mathrm{CH}) 2885-3047$, $v(\mathrm{C}=\mathrm{N}) 1605, v\left(\mathrm{C}=\mathrm{C}_{\text {ring }}\right) 1512, v(\mathrm{C}-\mathrm{O}) 1296, v(\mathrm{~V}=\mathrm{O}) 949, v(\mathrm{C}-\mathrm{Cl}) 720, v(\mathrm{C}-\mathrm{Br}) 648$. UV/Vis (DMSO), $\lambda_{\max }, \mathrm{nm}\left(\log \varepsilon, \mathrm{L} \mathrm{mol}^{-1} \mathrm{~cm}^{-1}\right): 263$ (4.7), 442 (4.11), 664 (1.9).

Typical procedure for the preparation of tetrahydro-4H-chromene derivatives (4a-g')

A mixture of dimedone (1a) or 1,3-cyclohexanedione (1b) (2 mmol), malononitrile (2) (2 $\mathrm{mmol})$, aromatic aldehydes (3a-r) $(2 \mathrm{mmol})$, and appropriate oxido-vanadium(IV) complex (10 $\mathrm{mol} \%)$ in EtOH $(10 \mathrm{~mL}$ ) was refluxed for the reported time in Table 5 (the progress of the reaction being monitored by TLC and hexane/ethyl acetate was used as an eluent). After completion of the reaction, the filtrate of the reaction mixture was achieved to recover oxidovanadium(IV) complexes and the reaction mixture was poured into ice-cold water; the crude product was filtered, dried and recrystallized from ethanol.

2-Amino-4-(2-bromophenyl)-7,7-dimethyl-5-oxo-5,6,7,8-tetrahydro-4H-chromene-3-carbonitrile (4f). Yellow powder; IR (KBr, $\left.v_{\max } / \mathrm{cm}^{-1}\right)$ : 3440, $3328\left(\mathrm{NH}_{2}\right), 2192(\mathrm{CN}), 1683(\mathrm{C}=\mathrm{O})$, 1590, $1542(\mathrm{C}=\mathrm{C}) .{ }^{1} \mathrm{H}$ NMR $\left(400 \mathrm{MHz}, \mathrm{DMSO}-\mathrm{d}_{6}\right) \delta_{\mathrm{ppm}}:$ 7.83-7.02 (m, 6H, CH-Ar, $\left.\mathrm{NH}_{2}\right), 4.69$ $(\mathrm{s}, 1 \mathrm{H}, \mathrm{CH}), 2.32\left(\mathrm{~d},{ }^{2} J_{H H}=8 \mathrm{~Hz}, \mathrm{CH}\right), 2.22\left(\mathrm{~d},{ }^{2} J_{H H}=8 \mathrm{~Hz}, \mathrm{CH}\right), 2.05\left(\mathrm{~d},{ }^{2} J_{H H}=8 \mathrm{~Hz}, \mathrm{CH}\right)$, $1.89\left(\mathrm{~d},{ }^{2} J_{H H}=8 \mathrm{~Hz}, \mathrm{CH}\right), 1.02\left(\mathrm{~s}, 3 \mathrm{H}, \mathrm{CH}_{3}\right), 0.96\left(\mathrm{~s}, 3 \mathrm{H}, \mathrm{CH}_{3}\right) .{ }^{13} \mathrm{C}$ NMR $\left(100 \mathrm{MHz}\right.$, DMSO-d $\left.\mathrm{d}_{6}\right)$ 
$\delta_{\mathrm{ppm}}: 197.72(\mathrm{C}=\mathrm{O}), 160.22,144.97,137.58,135.58,134.24,131.47,130.04,129.66,124.36$, $113.76(\mathrm{CN}), 70.00(\mathrm{C} 3), 58.80\left(\mathrm{CH}_{2}\right), 51.60\left(\mathrm{CH}_{2}\right), 36.67(\mathrm{CH}), 33.63\left(\mathrm{CMe}_{2}\right), 33.35\left(\mathrm{CH}_{3}\right)$, $30.05\left(\mathrm{CH}_{3}\right)$. Anal. calcd. for $\mathrm{C}_{18} \mathrm{H}_{17} \mathrm{BrN}_{2} \mathrm{O}_{2}: \mathrm{C}, 57.92 ; \mathrm{H}, 4.59 ; \mathrm{N}, 7.51 \%$. Found: $\mathrm{C}, 57.64 ; \mathrm{H}$, $4.37 ; \mathrm{N}, 7.24 \%$.

2-Amino-4-(furan-2-yl)-7,7-dimethyl-5-oxo-5, 6,7,8-tetrahydro-4H-chromene-3-carbonitrile (4p). Yellow powder; IR (KBr, $\left.v_{\max } / \mathrm{cm}^{-1}\right): 3408,3328\left(\mathrm{NH}_{2}\right), 2192(\mathrm{CN}), 1680(\mathrm{C}=\mathrm{O}), 1600$, $1555(\mathrm{C}=\mathrm{C}) .{ }^{\mathrm{H}} \mathrm{H}$ NMR $\left(400 \mathrm{MHz}, \mathrm{DMSO}-\mathrm{d}_{6}\right) \delta_{\mathrm{ppm}}: 7.46\left(\mathrm{~d},{ }^{3} J_{H H}=4 \mathrm{~Hz}, \mathrm{CH}-\mathrm{Ar}\right), 7.07(\mathrm{~s}, 2 \mathrm{H}$, $\left.\mathrm{NH}_{2}\right), 6.30-6.04(\mathrm{~m}, 2 \mathrm{H}, \mathrm{CH}-\mathrm{Ar}), 4.30(\mathrm{~s}, 1 \mathrm{H}, \mathrm{CH}), 2.47\left(\mathrm{~d},{ }^{2} J_{H H}=8 \mathrm{~Hz}, \mathrm{CH}\right), 2.40\left(\mathrm{~d},{ }^{2} J_{H H}=8\right.$ $\mathrm{Hz}, \mathrm{CH}), 2.27\left(\mathrm{~d},{ }^{2} J_{H H}=8 \mathrm{~Hz}, \mathrm{CH}\right), 2.15\left(\mathrm{~d},{ }^{2} J_{H H}=8 \mathrm{~Hz}, \mathrm{CH}\right), 1.03\left(\mathrm{~s}, 3 \mathrm{H}, \mathrm{CH}_{3}\right), 0.97(\mathrm{~s}, 3 \mathrm{H}$, $\left.\mathrm{CH}_{3}\right) .{ }^{13} \mathrm{C}$ NMR $\left(100 \mathrm{MHz}\right.$, DMSO-d $\left.\mathrm{d}_{6}\right) \delta_{\mathrm{ppm}}: 198.14(\mathrm{C}=\mathrm{O}), 163.22,160.92,157.34,143.35$, 135.21, 131.12, 123.78, $112.05(\mathrm{CN}), 77.68(\mathrm{C} 3), 57.01\left(\mathrm{CH}_{2}\right), 51.51\left(\mathrm{CH}_{2}\right), 33.41(\mathrm{CH}), 30.59$ $\left(\mathrm{CMe}_{2}\right), 30.02\left(\mathrm{CH}_{3}\right), 28.16\left(\mathrm{CH}_{3}\right)$. Anal. calcd. for $\mathrm{C}_{16} \mathrm{H}_{16} \mathrm{~N}_{2} \mathrm{O}_{3}: \mathrm{C}, 67.59 ; \mathrm{H}, 5.67 ; \mathrm{N}, 9.85 \%$. Found: C, 67.23; H, 5.45; N, 9.64\%.

2-Amino-7,7-dimethyl-5-oxo-4-(thiophen-2-yl)-5,6,7,8-tetrahydro-4H-chromene-3-carbonitrile $(4 q)$. White powder; IR (KBr, $\left.v_{\max } / \mathrm{cm}^{-1}\right)$ : 3392, $3328\left(\mathrm{NH}_{2}\right), 2192(\mathrm{CN}), 1673(\mathrm{C}=\mathrm{O}), 1596$, $1539(\mathrm{C}=\mathrm{C}) .{ }^{1} \mathrm{H}$ NMR $\left(400 \mathrm{MHz}, \mathrm{DMSO}-\mathrm{d}_{6}\right) \delta_{\mathrm{ppm}}: 7.30\left(\mathrm{~d},{ }^{3} J_{H H}=4 \mathrm{~Hz}, \mathrm{CH}-\mathrm{Ar}\right), 7.10(\mathrm{~s}, 2 \mathrm{H}$, $\left.\mathrm{NH}_{2}\right), 6.88-6.84(\mathrm{~m}, 2 \mathrm{H}, \mathrm{CH}-\mathrm{Ar}), 4.51(\mathrm{~s}, 1 \mathrm{H}, \mathrm{CH}), 2.45\left(\mathrm{~d},{ }^{2} J_{H H}=8 \mathrm{~Hz}, \mathrm{CH}\right), 2.40\left(\mathrm{~d},{ }^{2} J_{H H}=8\right.$ $\mathrm{Hz}, \mathrm{CH}), 2.28\left(\mathrm{~d},{ }^{2} J_{H H}=8 \mathrm{~Hz}, \mathrm{CH}\right), 2.13\left(\mathrm{~d},{ }^{2} J_{H H}=8 \mathrm{~Hz}, \mathrm{CH}\right), 1.02\left(\mathrm{~s}, 3 \mathrm{H}, \mathrm{CH}_{3}\right), 0.95(\mathrm{~s}, 3 \mathrm{H}$, $\left.\mathrm{CH}_{3}\right) .{ }^{13} \mathrm{C}$ NMR $\left(100 \mathrm{MHz}, \mathrm{DMSO}-\mathrm{d}_{6}\right) \delta_{\mathrm{ppm}}: 197.58(\mathrm{C}=\mathrm{O}), 164.10,160.54,150.88,128.41$, 125.99, 125.60, 121.20, $114.56(\mathrm{CN}), 68.42(\mathrm{C} 3), 59.73\left(\mathrm{CH}_{2}\right), 51.51\left(\mathrm{CH}_{2}\right), 33.33(\mathrm{CH}), 32.04$ $\left(\mathrm{CMe}_{2}\right), 30.25\left(\mathrm{CH}_{3}\right), 28.10\left(\mathrm{CH}_{3}\right)$. Anal. calcd. for $\mathrm{C}_{16} \mathrm{H}_{16} \mathrm{~N}_{2} \mathrm{O}_{2} \mathrm{~S}: \mathrm{C}, 63.98 ; \mathrm{H}, 5.37 ; \mathrm{N}, 9.33 \%$. Found: C, 63.72; H, 5.18; N, 9.14\%.

2-Amino-7,7-dimethyl-5-oxo-4-(pyridin-3-yl)-5,6,7,8-tetrahydro-4H-chromene-3-carbonitrile (4r). White powder; IR $\left(\mathrm{KBr}, v_{\max } / \mathrm{cm}^{-1}\right)$ : 3392, $3312\left(\mathrm{NH}_{2}\right), 2192(\mathrm{CN}), 1680(\mathrm{C}=\mathrm{O}), 1596$, $1574(\mathrm{C}=\mathrm{C}) .{ }^{1} \mathrm{H}$ NMR $\left(400 \mathrm{MHz}, \mathrm{DMSO}-\mathrm{d}_{6}\right) \delta_{\mathrm{ppm}}: 8.37\left(\mathrm{~s}, 2 \mathrm{H}, \mathrm{NH}_{2}\right), 7.52-7.10(\mathrm{~m}, 4 \mathrm{H}, \mathrm{CH}-$ Ar), $4.22(\mathrm{~s}, 1 \mathrm{H}, \mathrm{CH}), 2.47\left(\mathrm{~d},{ }^{2} J_{H H}=8 \mathrm{~Hz}, \mathrm{CH}\right), 2.39\left(\mathrm{~d},{ }^{2} J_{H H}=8 \mathrm{~Hz}, \mathrm{CH}\right), 2.22\left(\mathrm{~d},{ }^{2} J_{H H}=8 \mathrm{~Hz}\right.$, $\mathrm{CH}), 2.09\left(\mathrm{~d},{ }^{2} J_{H H}=8 \mathrm{~Hz}, \mathrm{CH}\right), 1.01\left(\mathrm{~s}, 3 \mathrm{H}, \mathrm{CH}_{3}\right), 0.92\left(\mathrm{~s}, 3 \mathrm{H}, \mathrm{CH}_{3}\right) .{ }^{13} \mathrm{C} \mathrm{NMR}(100 \mathrm{MHz}$, DMSO-d $\left._{6}\right) \delta_{\text {ppm }}: 197.33(\mathrm{C}=\mathrm{O}), 164.21,160.22,150.30,149.44,141.66,136.36,125.30,121.10$, $113.43(\mathrm{CN}), 67.08(\mathrm{C} 3), 58.98\left(\mathrm{CH}_{2}\right), 51.53\left(\mathrm{CH}_{2}\right), 35.04(\mathrm{CH}), 33.42\left(\mathrm{CMe}_{2}\right), 29.85\left(\mathrm{CH}_{3}\right)$, $28.52\left(\mathrm{CH}_{3}\right)$. Anal. calcd. for $\mathrm{C}_{17} \mathrm{H}_{17} \mathrm{~N}_{3} \mathrm{O}_{2}$ : $\mathrm{C}, 69.14 ; \mathrm{H}, 5.80 ; \mathrm{N}, 14.23 \%$. Found: $\mathrm{C}, 68.92 ; \mathrm{H}$, $5.63 ; \mathrm{N}, 14.04 \%$.

2-Amino-4-(4-bromophenyl)-5-oxo-5,6,7,8-tetrahydro-4H-chromene-3-carbonitrile (4w). White powder; IR $\left(\mathrm{KBr}, v_{\max } / \mathrm{cm}^{-1}\right): 3424,3344\left(\mathrm{NH}_{2}\right), 2192(\mathrm{CN}), 1680(\mathrm{C}=\mathrm{O}), 1593,1539(\mathrm{C}=\mathrm{C}) .{ }^{1} \mathrm{H}$ $\operatorname{NMR}\left(400 \mathrm{MHz}, \mathrm{DMSO}-\mathrm{d}_{6}\right) \delta_{\mathrm{ppm}}: 7.44\left(\mathrm{~d},{ }^{3} J_{H H}=4 \mathrm{~Hz}, \mathrm{CH}-\mathrm{Ar}\right), 7.10\left(\mathrm{~d},{ }^{3} J_{H H}=4 \mathrm{~Hz}, \mathrm{CH}-\mathrm{Ar}\right)$, $7.04\left(\mathrm{~s}, 2 \mathrm{H}, \mathrm{NH}_{2}\right), 4.15(\mathrm{~s}, 1 \mathrm{H}, \mathrm{CH}), 2.47-1.93\left(\mathrm{~m}, 6 \mathrm{H}, 3 \mathrm{CH}_{2}\right) \cdot{ }^{13} \mathrm{C}$ NMR $\left(100 \mathrm{MHz}, \mathrm{DMSO}-\mathrm{d}_{6}\right)$ $\delta_{\mathrm{ppm}}$ : $197.89(\mathrm{C}=\mathrm{O}), 166.05,160.06,145.82,132.79,131.10,124.54,121.20,114.92(\mathrm{CN})$, 59.24 (C3), $37.89(\mathrm{CH}), 36.70\left(\mathrm{CH}_{2}\right), 28.08\left(\mathrm{CH}_{2}\right), 21.37\left(\mathrm{CH}_{2}\right)$. Anal. calcd. for $\mathrm{C}_{16} \mathrm{H}_{13} \mathrm{BrN}_{2} \mathrm{O}_{2}$ : C, 55.67; H, 3.80; N, 8.12\%. Found: C, 55.48; H, 3.63; N, 7.91\%.

2-Amino-4-(2-bromophenyl)-5-oxo-5,6,7,8-tetrahydro-4H-chromene-3-carbonitrile (4x). Yellow powder; IR $\left(\mathrm{KBr}, v_{\max } / \mathrm{cm}^{-1}\right): 3472,3328\left(\mathrm{NH}_{2}\right), 2192(\mathrm{CN}), 1680(\mathrm{C}=\mathrm{O}), 1593,1545$ $(\mathrm{C}=\mathrm{C}) .{ }^{1} \mathrm{H}$ NMR $\left(400 \mathrm{MHz}, \mathrm{DMSO}-\mathrm{d}_{6}\right) \delta_{\mathrm{ppm}}: 7.51-7.08(\mathrm{~m}, 4 \mathrm{H}, \mathrm{CH}-\mathrm{Ar}), 7.05\left(\mathrm{~s}, 2 \mathrm{H}, \mathrm{NH}_{2}\right), 4.69$ (s, 1H, CH), 2.27-1.94 (m, 6H, 3CH $).{ }^{13} \mathrm{C}$ NMR (100 MHz, DMSO-d 6 ) $\delta_{\mathrm{ppm}}: 197.12(\mathrm{C}=\mathrm{O})$, $166.58,160.05,145.09,134.16,131.46,129.98,129.75,124.32,120.75,114.77(\mathrm{CN}), 58.67$ (C3), $37.93(\mathrm{CH}), 36.57\left(\mathrm{CH}_{2}\right), 28.10\left(\mathrm{CH}_{2}\right), 21.42\left(\mathrm{CH}_{2}\right)$. Anal. calcd. for $\mathrm{C}_{16} \mathrm{H}_{13} \mathrm{BrN}_{2} \mathrm{O}_{2}: \mathrm{C}$, 55.67 ; H, 3.80; N, 8.12\%. Found: C, 55.46; H, 3.65; N, 7.94\%. 
2-Amino-4-(2-nitrophenyl)-5-oxo-5,6,7,8-tetrahydro-4H-chromene-3-carbonitrile (4a'). Yellow powder; IR (KBr, $\left.v_{\max } / \mathrm{cm}^{-1}\right): 3408,3344\left(\mathrm{NH}_{2}\right), 2192(\mathrm{CN}), 1680(\mathrm{C}=\mathrm{O}), 1593,1523(\mathrm{C}=\mathrm{C}) .{ }^{1} \mathrm{H}$ NMR (400 MHz, DMSO-d $)_{6} \delta_{\text {ppm }}: 7.78-7.36(\mathrm{~m}, 4 \mathrm{H}, \mathrm{CH}-\mathrm{Ar}), 7.15\left(\mathrm{~s}, 2 \mathrm{H}, \mathrm{NH}_{2}\right), 4.91(\mathrm{~s}, 1 \mathrm{H}$, $\mathrm{CH}), 2.48-1.82\left(\mathrm{~m}, 6 \mathrm{H}, 3 \mathrm{CH}_{2}\right) .{ }^{13} \mathrm{C}$ NMR $\left(100 \mathrm{MHz}, \mathrm{DMSO}-\mathrm{d}_{6}\right) \delta_{\mathrm{ppm}}: 197.88(\mathrm{C}=\mathrm{O}), 166.22$, $160.66,150.54,140.59,134.99,132.01,129.38,125.24,120.73,114.86(\mathrm{CN}), 58.04$ (C3), 37.54 $(\mathrm{CH}), 31.73\left(\mathrm{CH}_{2}\right), 27.96\left(\mathrm{CH}_{2}\right), 21.30\left(\mathrm{CH}_{2}\right)$. Anal. calcd. for $\mathrm{C}_{16} \mathrm{H}_{13} \mathrm{~N}_{3} \mathrm{O}_{4}: \mathrm{C}, 61.73 ; \mathrm{H}, 4.21$; $\mathrm{N}, 13.50 \%$. Found: C, $61.54 ; \mathrm{H}, 4.04 ; \mathrm{N}, 13.33 \%$.

2-Amino-4-(furan-2-yl)-5-oxo-5,6,7,8-tetrahydro-4H-chromene-3-carbonitrile $\quad\left(4 \boldsymbol{e}^{\prime}\right) . \quad$ Brown powder; IR (KBr, $\left.v_{\max } / \mathrm{cm}^{-1}\right): 3408,3328\left(\mathrm{NH}_{2}\right), 2192(\mathrm{CN}), 1680(\mathrm{C}=\mathrm{O}), 1596,1587(\mathrm{C}=\mathrm{C}) .{ }^{1} \mathrm{H}$ NMR (400 MHz, DMSO-d $\left.)_{6}\right) \delta_{\mathrm{ppm}}: 7.45\left(\mathrm{~d},{ }^{3} J_{\mathrm{HH}}=4 \mathrm{~Hz}, \mathrm{CH}-\mathrm{Ar}\right), 7.05\left(\mathrm{~s}, 2 \mathrm{H}, \mathrm{NH}_{2}\right), 6.28-6.02$ $(\mathrm{m}, 2 \mathrm{H}, \mathrm{CH}-\mathrm{Ar}), 4.30(\mathrm{~s}, 1 \mathrm{H}, \mathrm{CH}), 2.47-1.93\left(\mathrm{~m}, 6 \mathrm{H}, 3 \mathrm{CH}_{2}\right) .{ }^{13} \mathrm{C}$ NMR $\left(100 \mathrm{MHz}, \mathrm{DMSO}-\mathrm{d}_{6}\right)$ $\delta_{\mathrm{ppm}}$ : $197.31(\mathrm{C}=\mathrm{O}), 166.75,160.88,157.41,143.37,121.01,113.07(\mathrm{CN}), 112.01,106.71$, $56.93(\mathrm{C} 3), 37.80(\mathrm{CH}), 30.58\left(\mathrm{CH}_{2}\right), 28.10\left(\mathrm{CH}_{2}\right), 21.36\left(\mathrm{CH}_{2}\right)$. Anal. calcd. for $\mathrm{C}_{14} \mathrm{H}_{12} \mathrm{~N}_{2} \mathrm{O}_{3}: \mathrm{C}$, 65.62 ; H, 4.72; N, 10.93\%. Found: C, 65.42; H, 4.56; N, 10.76\%.

2-Amino-5-oxo-4-(thiophen-2-yl)-5,6,7,8-tetrahydro-4H-chromene-3-carbonitrile $\left(4 f^{\prime}\right)$. White powder; IR (KBr, $\left.v_{\max } / \mathrm{cm}^{-1}\right): 3424,3344\left(\mathrm{NH}_{2}\right), 2192(\mathrm{CN}), 1680(\mathrm{C}=\mathrm{O}), 1596,1580(\mathrm{C}=\mathrm{C}) .{ }^{1} \mathrm{H}$ NMR (400 MHz, DMSO-d $\left.)_{6}\right) \delta_{\mathrm{ppm}}: 7.29\left(\mathrm{~d},{ }^{3} J_{\mathrm{HH}}=4 \mathrm{~Hz}, \mathrm{CH}-\mathrm{Ar}\right), 7.11\left(\mathrm{~s}, 2 \mathrm{H}, \mathrm{NH}_{2}\right), 6.88-6.83$ $(\mathrm{m}, 2 \mathrm{H}, \mathrm{CH}-\mathrm{Ar}), 4.51(\mathrm{~s}, 1 \mathrm{H}, \mathrm{CH}), 2.48-1.85\left(\mathrm{~m}, 6 \mathrm{H}, 3 \mathrm{CH}_{2}\right) .{ }^{13} \mathrm{C}$ NMR $\left(100 \mathrm{MHz}, \mathrm{DMSO}-\mathrm{d}_{6}\right)$ $\delta_{\mathrm{ppm}}: 197.02(\mathrm{C}=\mathrm{O}), 165.89,160.62,150.87,128.44,125.96,125.58,121.25,115.70(\mathrm{CN})$, $59.50(\mathrm{C} 3), 37.86(\mathrm{CH}), 31.94\left(\mathrm{CH}_{2}\right), 28.04\left(\mathrm{CH}_{2}\right), 21.37\left(\mathrm{CH}_{2}\right)$. Anal. calcd. for $\mathrm{C}_{14} \mathrm{H}_{12} \mathrm{~N}_{2} \mathrm{O}_{2} \mathrm{~S}$ : C, $61.75 ; \mathrm{H}, 4.44 ; \mathrm{N}, 10.29 \%$. Found: C, $61.54 ; \mathrm{H}, 4.27 ; \mathrm{N}, 10.10 \%$.

2-Amino-5-oxo-4-(pyridin-3-yl)-5,6,7,8-tetrahydro-4H-chromene-3-carbonitrile (4g'). White powder; IR (KBr, $\left.v_{\max } / \mathrm{cm}^{-1}\right): 3360,3312\left(\mathrm{NH}_{2}\right), 2192(\mathrm{CN}), 1664(\mathrm{C}=\mathrm{O}), 1580,1542(\mathrm{C}=\mathrm{C}) .{ }^{1} \mathrm{H}$ NMR (400 MHz, DMSO-d $)_{6} \delta_{\mathrm{ppm}}: 8.38\left(\mathrm{~s}, 2 \mathrm{H}, \mathrm{NH}_{2}\right), 7.53-7.09(\mathrm{~m}, 2 \mathrm{H}, \mathrm{CH}-\mathrm{Ar}), 4.21(\mathrm{~s}, 1 \mathrm{H}$, $\mathrm{CH}), 2.47-1.92\left(\mathrm{~m}, 6 \mathrm{H}, 3 \mathrm{CH}_{2}\right) .{ }^{13} \mathrm{C}$ NMR $\left(100 \mathrm{MHz}, \mathrm{DMSO}-\mathrm{d}_{6}\right) \delta_{\mathrm{ppm}}: 197.36(\mathrm{C}=\mathrm{O}), 166.48$, $160.15,150.29,149.38,141.71,136.36,125.23,121.14,114.40(\mathrm{CN}), 58.92(\mathrm{C} 3), 37.86(\mathrm{CH})$, $34.96\left(\mathrm{CH}_{2}\right), 28.11\left(\mathrm{CH}_{2}\right), 21.35\left(\mathrm{CH}_{2}\right)$. Anal. calcd. for $\mathrm{C}_{15} \mathrm{H}_{13} \mathrm{~N}_{3} \mathrm{O}_{2}: \mathrm{C}, 67.40 ; \mathrm{H}, 4.90 ; \mathrm{N}$, $15.72 \%$. Found: C, 67.19; H, 4.75; N, 15.53\%.

\section{RESULTS AND DISCUSSION}

General aspect

Schematic diagram for synthesis of [VO(L)(bipy)] and [VO(L)(phen)] complexes are shown in Scheme 2. These complexes were obtained in good yields of $67-71 \%$. They are partially soluble in common organic solvents, but have excellent solubility in DMF and DMSO. These complexes showed non-electrolyte behavior in DMSO $\left(10^{-3} \mathrm{M}\right)$.

\section{Spectral characterization}

Assignments of selected prominent IR bands in the $400-4000 \mathrm{~cm}^{-1}$ region for complexes are gathered in the Experimental section. In the FT-IR spectra of complexes, bands observed in the region of 2885-3448 $\mathrm{cm}^{-1}$ are assigned to $\mathrm{CH}$ vibrations. The bands at $948-949 \mathrm{~cm}^{-1}$ are due to $v(\mathrm{~V}=\mathrm{O})$ of the vanadyl moiety [35]. The stretching vibration of the $\mathrm{C}-\mathrm{O}$ bond is disclosed at 1288 and $1296 \mathrm{~cm}^{-1}$ for I and II, respectively [36]. The strong band at $1597 \mathrm{~cm}^{-1}$ is related to azomethine vibration of [VO(L)(bipy)] and it appeared at $1605 \mathrm{~cm}^{-1}$ in [VO(L)(phen)] [37].

In the electronic spectra of complexes, the bands at 276 and 263 can be related to a $\pi \rightarrow \pi^{*}$ transition. The bands appeared at $c a 444 \mathrm{~nm}$, are assigned to LMCT charge transfer. The Peaks at $658 \mathrm{~nm}$ in $\mathbf{I}$ and $664 \mathrm{~nm}$ in $\mathbf{I I}$ are related to $d-d$ transitions [36]. 


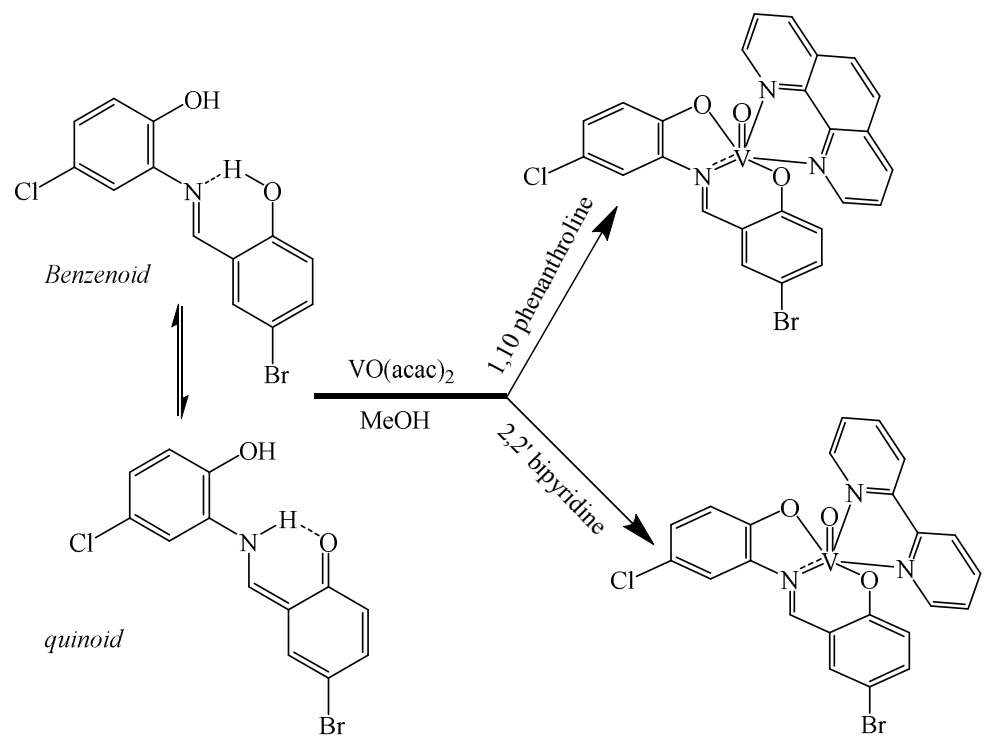

Scheme 2. Schematic diagram for the complexation process.

\section{Catalytic activity}

To optimize the reaction conditions, we used some polar and nonpolar solvents in the threecomponent reaction of dimedone 1a, malononitrile $\mathbf{2}$ and benzaldehyde 3a in the presence of catalysts such as $[\mathrm{VO}(\mathrm{L})($ phen $)]$ and $[\mathrm{VO}(\mathrm{L})($ bipy) $]$ as model reactions to investigate the effects of solvent for preparing compound 2-amino-7,7-dimethyl-5-oxo-4-phenyl-5,6,7,8-tetrahydro$4 H$-chromene-3-carbonitrile 4a. In each case, the substrates were mixed together with $10 \mathrm{~mol} \%$ $[\mathrm{VO}(\mathrm{L})(\mathrm{phen})]$ and $[\mathrm{VO}(\mathrm{L})($ bipy)$]$ agitated with $10 \mathrm{~mL}$ solvent under reflux. The results are shown in Table 2. The best results in terms of reaction time and yield of the desired product $\mathbf{4 a}$ was obtained when the reaction was conducted in ethanol.

We also optimized the quantity of catalysts. The best results were obtained when the reactions were carried out in the presence of $10 \mathrm{~mol} \%[\mathrm{VO}(\mathrm{L})(\mathrm{phen})]$ and $[\mathrm{VO}(\mathrm{L})(\mathrm{bipy})]$. The results are shown in Table 3.

We also attempted to reuse the catalysts by a variety of methods (Table 4). Direct reuse of the catalysts (Table 4, entry 2,6 ) led to a greater than $20 \%$ decrease in activity, while washing of the catalysts with dichloromethane and ethyl acetate, prior to reuse also resulted in lower conversions (Table 4, entry $3,4,7,8$ ). This phenomenon probably arose because the reactant and product were not completely desorbed from $[\mathrm{VO}(\mathrm{L})(\mathrm{phen})]$ and $[\mathrm{VO}(\mathrm{L})(\mathrm{bipy})]$ and therefore, the active sites were blocked. 
Table 2. Solvent effects on the three-component reaction of dimedone $\mathbf{1 a}$, malononitrile $\mathbf{2}$ and benzaldehyde $\mathbf{3 a}$.

\begin{tabular}{|c|c|c|c|c|}
\hline Entry & Solvent & Catalyst & Time (min) & Yield (\%) \\
\hline 1 & Ethanol & {$[\mathrm{VO}(\mathrm{L})(\mathrm{phen})]$} & 20 & 90 \\
\hline 2 & Ethyl acetate & {$[\mathrm{VO}(\mathrm{L})(\mathrm{phen})]$} & 35 & 85 \\
\hline 3 & Acetonitrile & {$[\mathrm{VO}(\mathrm{L})(\mathrm{phen})]$} & 40 & 83 \\
\hline 4 & Toluene & {$[\mathrm{VO}(\mathrm{L})(\mathrm{phen})]$} & 70 & 75 \\
\hline 5 & Ethanol & {$[\mathrm{VO}(\mathrm{L})(\mathrm{bipy})]$} & 35 & 88 \\
\hline 6 & Ethyl acetate & {$[\mathrm{VO}(\mathrm{L})($ bipy)] } & 50 & 83 \\
\hline 7 & Acetonitrile & {$[\mathrm{VO}(\mathrm{L})($ bipy) } & 60 & 80 \\
\hline 8 & Toluene & {$[\mathrm{VO}(\mathrm{L})($ bipy) $)$} & 90 & 70 \\
\hline
\end{tabular}

Table 3. Optimized the quantity of catalysts on the three-component reaction for the synthesis of $\mathbf{4 a}$.

\begin{tabular}{|c|c|c|c|c|}
\hline Entry & Catalyst & Mol\% catalyst & Time (min) & Yield (\%) \\
\hline 1 & {$[\mathrm{VO}(\mathrm{L})($ phen $)]$} & 5 & 28 & 83 \\
\hline 2 & {$[\mathrm{VO}(\mathrm{L})($ bipy)$)]$} & 5 & 50 & 80 \\
\hline 3 & {$[\mathrm{VO}(\mathrm{L})($ phen $)]$} & 7.5 & 23 & 88 \\
\hline 4 & {$[\mathrm{VO}(\mathrm{L})($ bipy) $]$} & 7.5 & 40 & 85 \\
\hline 5 & {$[\mathrm{VO}(\mathrm{L})($ phen) $]$} & 10 & 20 & 90 \\
\hline 6 & {$[\mathrm{VO}(\mathrm{L})($ bipy) } & 10 & 35 & 88 \\
\hline 7 & {$[\mathrm{VO}(\mathrm{L})($ phen) $)$} & 12.5 & 20 & 90 \\
\hline 8 & {$[\mathrm{VO}(\mathrm{L})($ bipy) } & 12.5 & 35 & 88 \\
\hline
\end{tabular}

Table 4. Reusability of $[\mathrm{VO}(\mathrm{L})(\mathrm{phen})]$ and $[\mathrm{VO}(\mathrm{L})($ bipy $)]$ on the three-component reaction for the synthesis of $4 \mathbf{a}$.

\begin{tabular}{|c|c|c|c|}
\hline Entry & Catalyst & Time $(\min )$ & Yield (\%) \\
\hline 1 & {$[\mathrm{VO}(\mathrm{L})(\mathrm{phen})]\left(1^{\mathrm{st}}\right.$ use $)$} & 20 & 90 \\
\hline 2 & {$[\mathrm{VO}(\mathrm{L})(\mathrm{phen})]^{\mathrm{a}}\left(2^{\mathrm{st}}\right.$ use $)$} & 40 & 70 \\
\hline 3 & {$[\mathrm{VO}(\mathrm{L})(\mathrm{phen})]^{\mathrm{b}}\left(2^{\mathrm{st}}\right.$ use $)$} & 28 & 85 \\
\hline 4 & {$[\mathrm{VO}(\mathrm{L})(\mathrm{phen})]^{\mathrm{c}}\left(2^{\mathrm{st}}\right.$ use $)$} & 30 & 83 \\
\hline 5 & {$\left[\mathrm{VO}(\mathrm{L})\right.$ (bipy)] (1 ${ }^{\mathrm{st}}$ use) } & 35 & 88 \\
\hline 6 & {$[\mathrm{VO}(\mathrm{L})(\mathrm{bipy})]^{\mathrm{a}}\left(2^{\mathrm{st}}\right.$ use $)$} & 60 & 70 \\
\hline 7 & {$[\mathrm{VO}(\mathrm{L})(\mathrm{bipy})]^{\mathrm{b}}\left(2^{\mathrm{st}}\right.$ use $)$} & 45 & 83 \\
\hline 8 & {$[\mathrm{VO}(\mathrm{L})(\text { bipy })]^{\mathrm{c}}\left(2^{\mathrm{st}}\right.$ use $)$} & 48 & 80 \\
\hline
\end{tabular}

${ }^{\mathrm{a} D i r e c t}$ reuse of the catalysts. ${ }^{\mathrm{b}}[\mathrm{VO}(\mathrm{L})($ phen $)]$ and $[\mathrm{VO}(\mathrm{L})($ bipy $)]$ were washed with dichloromethane. ${ }^{c}[\mathrm{VO}(\mathrm{L})($ phen $)]$ and $[\mathrm{VO}(\mathrm{L})($ bipy $)]$ were washed with ethylacetate.

To rely on our collected data, we decided to apply this method for synthesis of tetrahydrobenzopyran derivatives by using three-component reaction of dimedone (1a) or 1,3cyclohexanedione (1b), malononitrile (2) and aromatic aldehydes (3a-r), in EtOH, at reflux, in the presence of $10 \mathrm{~mol} \%[\mathrm{VO}(\mathrm{L})(\mathrm{phen})]$ and $[\mathrm{VO}(\mathrm{L})($ bipy) $]$ (Table 5). 
Table 5. Three-component reaction of dimedone (1a) or 1,3-cyclohexanedione (1b), malononitrile (2) and aromatic aldehydes (3a-r).

\begin{tabular}{|c|c|c|c|c|c|c|c|c|}
\hline \multirow{2}{*}{$\begin{array}{l}\text { Compd. } \\
\text { No. }\end{array}$} & \multirow[t]{2}{*}{$\mathrm{R}$} & \multirow[t]{2}{*}{$\mathrm{Ar}$} & \multicolumn{2}{|c|}{$[\mathrm{VO}(\mathrm{L})($ phen $)]$} & \multicolumn{2}{|c|}{ [VO(L)(bipy)] } & \multirow{2}{*}{$\begin{array}{c}\text { M.P. } \\
\text { observed }\left({ }^{\circ} \mathrm{C}\right)\end{array}$} & \multirow{2}{*}{$\begin{array}{c}\text { M.P. } \\
\text { reported }\left({ }^{\circ} \mathrm{C}\right)\end{array}$} \\
\hline & & & $\begin{array}{l}\text { Time } \\
(\mathrm{min})\end{array}$ & $\begin{array}{l}\text { Yield } \\
(\%)\end{array}$ & $\begin{array}{l}\text { Time } \\
(\mathrm{min})\end{array}$ & $\begin{array}{c}\text { Yield } \\
(\%)\end{array}$ & & \\
\hline $4 \mathrm{a}$ & $\mathrm{Me}$ & $\mathrm{C}_{6} \mathrm{H}_{5}$ & 20 & 90 & 35 & 88 & $231-232$ & $234-236$ [19] \\
\hline $4 \mathrm{~b}$ & $\mathrm{Me}$ & $4-\mathrm{Cl}-\mathrm{C}_{6} \mathrm{H}_{4}$ & 17 & 91 & 32 & 90 & $215-217$ & $216-218[19]$ \\
\hline $4 c$ & $\mathrm{Me}$ & $2-\mathrm{Cl}-\mathrm{C}_{6} \mathrm{H}_{4}$ & 18 & 91 & 33 & 89 & $210-212$ & $214-215$ [19] \\
\hline $4 d$ & $\mathrm{Me}$ & $2,4-(\mathrm{Cl})_{2}-\mathrm{C}_{6} \mathrm{H}_{3}$ & 15 & 92 & 30 & 91 & $175-178$ & $178-179[21]$ \\
\hline $4 \mathrm{e}$ & $\mathrm{Me}$ & $4-\mathrm{Br}-\mathrm{C}_{6} \mathrm{H}_{4}$ & 16 & 90 & 33 & 89 & $221-222$ & $222-224[20]$ \\
\hline $4 \mathrm{f}$ & $\mathrm{Me}$ & $2-\mathrm{Br}-\mathrm{C}_{6} \mathrm{H}_{4}$ & 17 & 90 & 32 & 89 & $108-110$ & \\
\hline $4 \mathrm{~g}$ & $\mathrm{Me}$ & $4-\mathrm{F}-\mathrm{C}_{6} \mathrm{H}_{4}$ & 16 & 91 & 32 & 90 & $207-210$ & $208-211[19]$ \\
\hline $4 \mathrm{~h}$ & $\mathrm{Me}$ & $4-\mathrm{NO}_{2}-\mathrm{C}_{6} \mathrm{H}_{4}$ & 15 & 91 & 30 & 90 & 181-182 & $180-182[19]$ \\
\hline $4 \mathrm{i}$ & $\mathrm{Me}$ & $3-\mathrm{NO}_{2}-\mathrm{C}_{6} \mathrm{H}_{4}$ & 17 & 90 & 33 & 89 & $213-216$ & $217-219[19]$ \\
\hline $4 j$ & $\mathrm{Me}$ & $2-\mathrm{NO}_{2}-\mathrm{C}_{6} \mathrm{H}_{4}$ & 16 & 91 & 32 & 90 & $214-217$ & $215-217[21]$ \\
\hline $4 \mathrm{k}$ & $\mathrm{Me}$ & $4-\mathrm{CH}_{3}-\mathrm{C}_{6} \mathrm{H}_{4}$ & 23 & 89 & 38 & 86 & $218-220$ & $219-221[19]$ \\
\hline 41 & $\mathrm{Me}$ & $4-\mathrm{CH}_{3} \mathrm{O}-\mathrm{C}_{6} \mathrm{H}_{4}$ & 25 & 88 & 40 & 85 & $200-202$ & 201-203 [19] \\
\hline $4 m$ & $\mathrm{Me}$ & $2-\mathrm{CH}_{3} \mathrm{O}-\mathrm{C}_{6} \mathrm{H}_{4}$ & 23 & 90 & 38 & 85 & $231-233$ & $231-232[20]$ \\
\hline $4 n$ & $\mathrm{Me}$ & $4-\mathrm{OH}-\mathrm{C}_{6} \mathrm{H}_{4}$ & 27 & 87 & 45 & 83 & $225-228$ & $226-228[19]$ \\
\hline 40 & $\mathrm{Me}$ & $4-\left(\mathrm{CH}_{3}\right)_{2}-\mathrm{N}-\mathrm{C}_{6} \mathrm{H}_{4}$ & 28 & 87 & 45 & 82 & $208-210$ & $210-212$ [19] \\
\hline $4 p$ & $\mathrm{Me}$ & Furan-2-yl & 23 & 88 & 38 & 85 & $221-222$ & $220-222[19]$ \\
\hline $4 q$ & $\mathrm{Me}$ & Thiophen-2-yl & 25 & 88 & 40 & 84 & $227-229$ & $226-228[19]$ \\
\hline $4 \mathrm{r}$ & $\mathrm{Me}$ & Pyridin-3-yl & 20 & 89 & 35 & 87 & $206-207$ & - \\
\hline $4 \mathrm{~s}$ & $\mathrm{H}$ & $\mathrm{C}_{6} \mathrm{H}_{5}$ & 22 & 89 & 38 & 86 & $230-231$ & $229-231[21]$ \\
\hline $4 \mathrm{t}$ & $\mathrm{H}$ & $4-\mathrm{Cl}-\mathrm{C}_{6} \mathrm{H}_{4}$ & 17 & 90 & 35 & 89 & $223-226$ & $225-227[21]$ \\
\hline $4 \mathrm{u}$ & $\mathrm{H}$ & $2-\mathrm{Cl}-\mathrm{C}_{6} \mathrm{H}_{4}$ & 18 & 90 & 35 & 89 & 195-197 & $197-199[20]$ \\
\hline $4 \mathrm{v}$ & $\mathrm{H}$ & $2,4-(\mathrm{Cl})_{2}-\mathrm{C}_{6} \mathrm{H}_{3}$ & 16 & 91 & 32 & 90 & $220-222$ & $221-223[21]$ \\
\hline $4 w$ & $\mathrm{H}$ & $4-\mathrm{Br}-\mathrm{C}_{6} \mathrm{H}_{4}$ & 18 & 90 & 35 & 88 & $248-250$ & \\
\hline $4 \mathrm{x}$ & $\mathrm{H}$ & $2-\mathrm{Br}-\mathrm{C}_{6} \mathrm{H}_{4}$ & 20 & 89 & 37 & 87 & $215-217$ & - \\
\hline $4 y$ & $\mathrm{H}$ & $4-\mathrm{NO}_{2}-\mathrm{C}_{6} \mathrm{H}_{4}$ & 16 & 91 & 32 & 90 & $234-237$ & $235-237[21]$ \\
\hline $4 z$ & $\mathrm{H}$ & $3-\mathrm{NO}_{2}-\mathrm{C}_{6} \mathrm{H}_{4}$ & 19 & 89 & 35 & 88 & $200-202$ & $201-203[21]$ \\
\hline $4 \mathrm{a}^{\prime}$ & $\mathrm{H}$ & $2-\mathrm{NO}_{2}-\mathrm{C}_{6} \mathrm{H}_{4}$ & 17 & 91 & 33 & 90 & $200-202$ & - \\
\hline $4 \mathrm{~b}^{\prime}$ & $\mathrm{H}$ & $4-\mathrm{CH}_{3}-\mathrm{C}_{6} \mathrm{H}_{4}$ & 25 & 89 & 40 & 86 & $223-225$ & $224-226[20]$ \\
\hline $4 \mathrm{c}^{\prime}$ & $\mathrm{H}$ & $4-\mathrm{CH}_{3} \mathrm{O}-\mathrm{C}_{6} \mathrm{H}_{4}$ & 27 & 88 & 43 & 85 & 189-191 & $190-192[21]$ \\
\hline $4 \mathrm{~d}^{\prime}$ & $\mathrm{H}$ & $4-\mathrm{OH}-\mathrm{C}_{6} \mathrm{H}_{4}$ & 30 & 87 & 50 & 83 & $256-258$ & $257-259[20]$ \\
\hline $4 \mathrm{e}^{\prime}$ & $\mathrm{H}$ & Furan-2-yl & 25 & 88 & 45 & 84 & $237-239$ & - \\
\hline $4 f^{\prime}$ & $\mathrm{H}$ & Thiophen-2-yl & 27 & 87 & 48 & 83 & $210-211$ & $=$ \\
\hline $4 \mathrm{~g}^{\prime}$ & $\mathrm{H}$ & Pyridin-3-yl & 22 & 89 & 42 & 85 & $229-230$ & \\
\hline
\end{tabular}

In these reactions, in first step Knoevenagel condensation takes place to form the 2arylidenemalononitrile derivatives (A). The active methine of cyclic $\beta$-dicarbonyl compounds react with the electrophilic $\mathrm{C}=\mathrm{C}$ double bond of 2-arylidenemalononitrile to give the intermediate (B) which tautomerizes into $\mathbf{C}$. The next step is cyclized by nucleophilic attack of the negative oxygen atom on the cyano $(\mathrm{CN})$ moiety, giving intermediate $\mathbf{D}$. The expected product $\mathbf{4 a - 4 g ^ { \prime }}$ (tetrahydrobenzopyrans), is afforded by tautomerization. Here, the catalytic activity of the complexes was established for Knoevenagel condensation and Michael addition reactions. Whereas $\mathrm{V}^{5+}$ as a Lewis acid, coordinated to the oxygen of carbonyl group of aldehyde and nitrogen of cyano $(\mathrm{CN})$ moiety, polarized the $\pi$-electron cloud carbonyl and cyano $(\mathrm{CN})$ group, which leads to attack by nucleophiles easier to carbonyl group and cyano $(\mathrm{CN})$ moiety (Scheme 3). 
<smiles>[R]C1([R])CC(=O)C2=C(C1)OC(N)=C(C#N)C2[Al]</smiles>

Tetrahydrobenzopyran derinatives (4a-g')

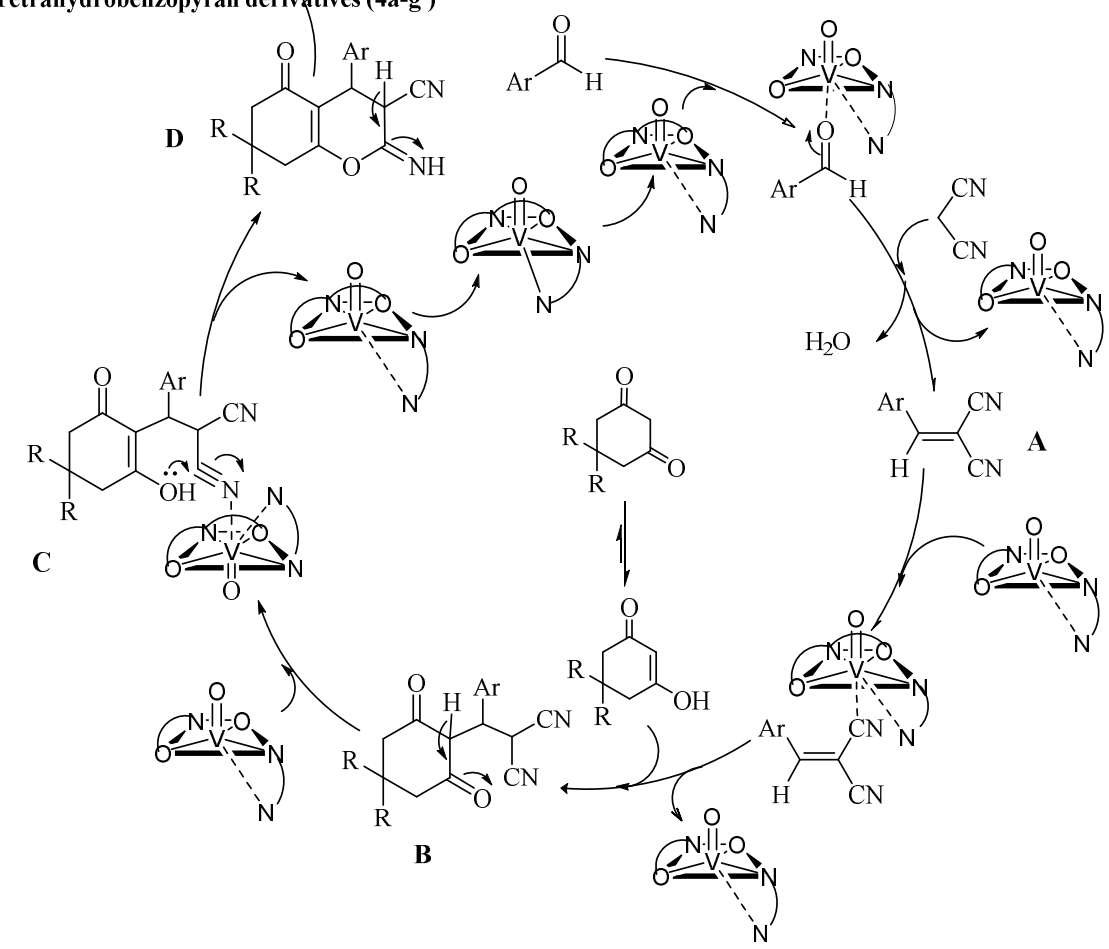

Scheme 3. Proposed mechanism for the synthesis of tetrahydrobenzopyran derivatives catalyzed by oxido-vanadium(IV) complexes (I and II).

\section{CONCLUSION}

The present study reports, synthesis and characterization of two mixed ligand oxidovanadium(IV) complexes containing ONO tridentate Schiff base, 4-bromo-2-(( 5-chloro-2hydroxyphenyl)imino)methyl)phenol $\left(\mathrm{H}_{2} \mathrm{~L}\right)$ and appropriate aromatic hetrocylic base, 2,2'bipyridine and 1,10-phenanthroline for $[\mathrm{VO}(\mathrm{L})($ bipy $)]$ and $[\mathrm{VO}(\mathrm{L})(\mathrm{phen})]$, respectively. We have also investigated the use of these complexes as new and effective catalysts, for threecomponent reaction of dimedone or 1,3-cyclohexanedione, malononitrile and aromatic aldehydes, which leads to the synthesis of tetrahydrobenzopyran derivatives. These reactions were carried out in EtOH at reflux. High yields, clean reaction conditions in comparison with existing methods and reusability of the catalyst with consistent activity are advantages of this procedure that make it a useful practical process for the synthesis of these compounds.

\section{ACKNOWLEDGMENTS}

The authors express their great appreciation to Pharmaceutics Research Center, Institute of Neuropharmacology, Kerman University of Medical Sciences for supporting this investigation. 


\section{REFERENCES}

1. Zhu, J. Multicomponent Reactions, Wiley-VCH: Weinheim; 2005.

2. Tietze, L.F. Chem. Rev. 1996, 96, 115.

3. Baghernejad, B. Bull. Chem. Soc. Ethiop. 2014, 28, 149.

4. Hussain, A.M.; Mansoor, S.S.; Aswin, K.; Logaiya, K.; Sudhan, S.P.N. Bull. Chem. Soc. Ethiop. 2014, 28, 91.

5. Karimi-Jaberi, Z.; Fakhraei, H. Bull. Chem. Soc. Ethiop. 2012, 26, 473.

6. Heravi, M.M.; Alinejhad, H.; Bakhtiari, K.; Saeedi, M.; Oskooie, H.A.; Bamoharram, F.F. Bull. Chem. Soc. Ethiop. 2011, 25, 399.

7. Rafiee, E.; Rashidzadeh, S.; Eavani, S.; Joshaghani, M. Bull. Chem. Soc. Ethiop. 2010, 24, 209.

8. Foye, W.O. Principi di Chimica Farmaceutica, II, Piccin: Padova, Italy; 1991, p 416.

9. Mohr, S.J.; Chirigos, M.A.; Fuhrman, F.S.; Pryor, J.W. Cancer Res. 1975, 35, 3750.

10. Davoll, J.; Clarke, J.; Eislager, E.F. J. Med. Chem. 1972, 15, 837.

11. Shwetaa, T.N.; Gupta, S. Bioorg. Med. Chem. Lett. 2004, 14, 3913.

12. El-Agrody, A.M; El-Hakium, M.H.; Abd El-Latif, M.S.; Fekry, A.H.; El-Sayed, E.S.M.; ElGareab, K.A. Acta. Pharm. 2000, 50, 111.

13. Ahluwalia, V.K.; Batla, R.; Khurana, A.; Kumar, R. Indian J. Chem. Sec. B. 1990, 29, 1141.

14. Wang, J.L.; Liu, D.; Zhang, Z.J.; Shan, S.; Han, X.; Srinivasula, S.M.; Croce, C.M.; Alnemri, E.S.; Huang, Z. Proc. Natl. Acad. Sci. USA 2007, 97, 7124.

15. Bonsignore, L.; Loy, G.; Secci, D.; Calignano, A. Eur. J. Med. Chem. 1993, $28,517$.

16. Kidwai, M.; Saxena, S.; Khan, M.K.R.; Thukral, S.S. Bioorg. Med. Chem. Lett. 2005, 15, 4295.

17. Heber, D.; Heers, C.; Ravens, U. Pharmazie, 1993, 48, 537.

18. Konkoy, C.S.; Fick, D.B.; Cai, S.X.; Lan, N.C.; Keana, J.F.W. PCT. Int. Appl. WO. 2000, 0075123.

19. Dekamin, M.G.; Eslami, M.; Maleki, A. Tetrahedron 2013, 69, 1074.

20. Safaei, H.R.; Shekouhy, M.; Shirinfeshan, A.; Rahmanpur, S. Mol. Divers. 2012, 16, 669.

21. Seifi, M.; Sheibani, H. Catal. Lett. 2008, 126, 275.

22. Jin, T.S.; Xiao, J.C.; Wang, S.J.; Li, T.S.; Song, X.R. Synlett 2003, 2001.

23. Hosseini-Sarvari, M.; Shafiee-Haghighi, S. Chem. Heterocycl. Compd. 2012, 48, 1307.

24. Zhou, Z.; Yang, F.; Wu, L.; Zhang, A. Chem. Sci. Trans. 2012, 1, 57.

25. Niralwad, K.S.; Shelke, K.F.; Sadaphal, S.S.; Shingate, B.B.; Shingare, M.S. Bull. Catal. Soc. India 2009, 8, 188.

26. Shia, D.; Moua, J.; Zhuanga, Q.; Wang, X. J. Chem. Res. 2004, 821.

27. Boumoud, B.; Yahiaoui, A.A.; Boumoud, T.; Debache, A. J. Chem. Pharm. Res. 2012, 4, 795.

28. Khurana, M.J.; Nand, B.; Saluja, P. Tetrahedron, 2010, 66, 5637.

29. Abaszadeh, M.; Seifi, M.; Asadipour, A. Res. Chem. Intermed. 2015, 41, 5229.

30. Abaszadeh, M.; Seifi, M. Res. Chem. Intermed. 2015, 41, 7715.

31. Amirheidari, B.; Seifi, M.; Abaszadeh, M. Res. Chem. Intermed. 2015, doi: 10.1007/s11164015-2220-1.

32. Abaszadeh, M.; Seifi, M. Lett. Org. Chem. 2015, 12, 271.

33. Takjoo, R.; Mague, T.; Akbari, A.; Ebrahimipour, S.Y. J. Coord. Chem. 2013, 66, 2852.

34. Ebrahimipour, S.Y.; Mague, T.; Akbari, A.; Takjoo, R. J. Mol. Struct. 2012, 1028, 148.

35. Noshiranzadeh, N.; Mayeli, M.; Bikas, R.; Slepokura, K.; Lis, T. Transit. Met. Chem. 2014, $39,33$.

36. Ebrahimipour, S.Y.; Sheikhshoaie, I.; Crochet, A.; Khaleghi, M.; Fromm, K. J. Mol. Struct. 2014, 1072, 267.

37. Zhai, S.; Guo, Q.; Dong, J.; Xu, T.; Li, L. Transit. Met. Chem. 2014, 39, 271. 